\title{
Focus on ethics and palliative care in the intensive care unit
}

\author{
Katherine R. Courtright ${ }^{1,2}$, Dominique D. Benoit ${ }^{3}$ and J. Randall Curtis ${ }^{4,5^{*}}$
}

(c) 2019 Springer-Verlag GmbH Germany, part of Springer Nature

This focus editorial highlights papers on prognostic and palliative care strategies for critically ill patients and their families that were published in Intensive Care Medicine (ICM) and other journals in the last 2 years, including five original research papers, one systematic review, one pragmatic review, six "what's new", two "understanding the disease", and one editorial.

The past several decades of critical care research have led to numerous treatment and technological advances resulting in improved ICU survival, but interventions to improve patient- and family-centered care have not kept pace $[1,2]$. However, emerging literature suggests there is a renewed focus on comprehensive approaches to critical care grounded in partnerships between patients, families, and healthcare professionals. A key component of such efforts is highlighted in a recently published ICM editorial that provides ten evidence-based principles of palliative care in the ICU [3]. This framework builds on the recognition that the highest quality critical care is provided simultaneously with, not independently from, palliative care for all critically ill patients to address symptoms, communication about goals of care, family support, shared decision-making, and in some cases, dying. ICU clinicians are responsible for providing primary or basic palliative care in all of these domains, yet often lack the education and training to do so. Having a high-quality discussion about poor prognosis with patients and families remains one of the most daunting tasks for ICU clinicians [4], and has been further complicated by an increasing need to incorporate diverse

\footnotetext{
*Correspondence: jrc@u.washington.edu

${ }^{5}$ Division of Pulmonary, Critical Care, and Sleep Medicine, Harborview Medical Center, University of Washington, 325 Ninth Avenue, Box 359762 , Seattle, WA 98104, USA

Full author information is available at the end of the article
}

cultural beliefs, values, and attitudes around serious illness, dying, and death in our increasingly multi-cultural societies [5]. There are many online resources and inperson workshops available to support ICU clinicians' education in primary palliative care, and opportunities to measure the impact of such interventions on the quality of patient- and family-centered outcomes [3].

An improved understanding of family-centered care in the ICU will advance research and policy efforts to reduce the psychological burdens frequently experienced by families during and after critical illness of a loved one. A summary of the latest clinical practice guideline for family-centered care in the ICU highlights the importance of pursuing such efforts, but also notes that all 23 recommendations were graded as weak based on the quality of current evidence [6]. There has since been a high-quality stepped-wedge, cluster-randomized trial that describes the impact of a multicomponent familysupport intervention among surrogates of 1420 critically ill patients across five ICUs [7]. The intervention was led by nurses and involved daily communication with families and protocolized clinician-family meetings throughout the ICU course. This intervention resulted in a reduced ICU length of stay and improvements in surrogates' perception of the quality of communication and the patient- and family-centeredness of care, but did not impact surrogates' symptoms of depression or posttraumatic stress after the ICU. Assessing families' psychological outcomes after the ICU has proven to be a pervasive challenge due, at least in part, to a persistent difficulty in detecting responsiveness of the scales used to assess such outcomes [8]. However, a recent large quasi-experimental study conducted across 9 ICUs tested the impact of an informational brochure and website for families showing a significant reduction in posttraumatic stress symptoms using the Short Screening Scale for Posttraumatic Stress Disorder [9]. Although these two studies represent

\section{Springer}


promising advancements in the state of the science for family-centered care, we need a deeper understanding of factors that increase risk for adverse psychological outcomes.

Another innovative area of ICU research focuses on the very elderly ( $\geq 80$ years of age) and specifically, the need to identify patients in this rapidly growing subgroup who are most likely to benefit from ICU care [10]. Several recent observational studies have challenged the common view that very elderly patients gain little survival or quality of life benefit from ICU admission [11]. One such study conducted among 31 Dutch ICUs and more than 200,000 patients, with $13 \%$ being $\geq 80$ years of age, found that annual risk-adjusted short- and long-term mortality rates significantly decreased between 2008 and 2014 for both the very elderly patients and those aged $<80$ years [12]. Inherent limitations of retrospective research from a single country notwithstanding, such results suggest that improvements in ICU care over time may benefit patients regardless of age and further examination of other risk factors for poor outcomes is needed. Moreover, a recent conceptual model of the policy, market, hospital, clinician, patient, and family factors that influence the decision to admit elderly patients to the ICU provides an important framework for future work to understand and address the undue variability in this population's care [13]. An expert-based research agenda highlights these and other major evidence gaps for critically ill elderly patients and suggests several high-quality studies that would improve patient- and family-centered care before, during, and after ICU admission for very elderly patients, as well as inform triage practices during times of capacity strain [14].

Frailty and other pre-ICU functional status indicators have gained attention as potentially better predictors of poor outcomes than age or traditional ICU scoring systems [11]. A recent systematic review of ten observational studies including 3030 critically ill adults found that $30 \%$ were frail at baseline and that frail patients experienced higher rates of hospital and long-term mortality and lower rates of discharge to home compared to fit patients [15]. Studies summarized in this systematic review provide important insights into an extremely vulnerable ICU population and underscore the need for hospitals and ICUs to incorporate standardized frailty and functional status assessments into the admission process in order to promote continued research and clinical efforts in this area. In the next couple of years, we need to find better ways to identify patients and family members at high risk for poor outcomes, especially among the growing population of elderly in our societies.

\section{Author details}

${ }^{1}$ Department of Medicine, Perelman School of Medicine, University of Pennsylvania, Philadelphia, USA. ${ }^{2}$ Palliative and Advanced IIIness Research (PAIR) Center, Perelman School of Medicine, University of Pennsylvania, Philadelphia, USA. ${ }^{3}$ Department of Intensive Care Medicine, Ghent University Hospital, Ghent, Belgium. ${ }^{4}$ Cambia Palliative Center of Excellence at UW Medicine, Seattle, WA, USA. ${ }^{5}$ Division of Pulmonary, Critical Care, and Sleep Medicine, Harborview Medical Center, University of Washington, 325 Ninth Avenue, Box 359762, Seattle, WA 98104, USA.

\section{Compliance with ethical standards}

\section{Conflicts of interest}

The authors have no conflicts.

\section{Ethical approval}

An approval by an ethics committee was not applicable.

\section{Publisher's Note}

Springer Nature remains neutral with regard to jurisdictional claims in published maps and institutional affiliations.

Received: 11 February 2019 Accepted: 14 March 2019

Published online: 25 March 2019

References

1. La Calle GH, Ovies AA, Tello VG (2017) A plan for improving the humanisation of intensive care units. Intensive Care Med 43(4):547-549

2. Davidson JE, Aslakson RA, Long AC et al (2017) Guidelines for familycentered care in the neonatal, pediatric, and adult ICU. Crit Care Med 45(1):103-128

3. Edwards JD, Voigt LP, Nelson JE (2017) Ten key points about ICU palliative care. Intensive Care Med 43(1):83-85

4. Isaac M, Curtis JR (2017) How to respond to an ICU patient asking if she/ he is going to die. Intensive Care Med 43(2):220-222

5. Bein T (2017) Understanding intercultural competence in intensive care medicine. Intensive Care Med 43(2):229-231

6. Gerritsen RT, Hartog CS, Curtis JR (2017) New developments in the provision of family-centered care in the intensive care unit. Intensive Care Med 43(4):550-553

7. White DB, Angus DC, Shields AM et al (2018) A randomized trial of a family-support intervention in intensive care units. N Engl J Med 378(25):2365-2375

8. Courtright KR, Benoit DD, Halpern SD (2017) Life after death in the ICU: detecting family-centered outcomes remains difficult. Intensive Care Med 43(10):1529-1531

9. Mistraletti G, Umbrello M, Mantovani ES et al (2017) A family information brochure and dedicated website to improve the ICU experience for patients' relatives: an Italian multicenter before-and-after study. Intensive Care Med 43(1):69-79

10. Leblanc G, Boumendil A, Guidet B (2017) Ten things to know about critically ill elderly patients. Intensive Care Med 43(2):217-219

11. Robert R, Skrifvars MB, Ranzani OT (2017) Is this critically ill patient elderly or too old? Intensive Care Med 43(12):1884-1886

12. Karakus A, Haas LEM, Brinkman S, de Lange DW, de Keizer NF (2017) Trends in short-term and 1-year mortality in very elderly intensive care patients in the Netherlands: a retrospective study from 2008 to 2014. Intensive Care Med 43(10):1476-1484

13. Curtis JR, Engelberg RA, Teno JM (2017) Understanding variability of endof-life care in the ICU for the elderly. Intensive Care Med 43(1):94-96

14. Flaatten $\mathrm{H}$, de Lange DW, Artigas A et al (2017) The status of intensive care medicine research and a future agenda for very old patients in the ICU. Intensive Care Med 43(9):1319-1328

15. Muscedere J, Waters B, Varambally A et al (2017) The impact of frailty on intensive care unit outcomes: a systematic review and meta-analysis. Intensive Care Med 43(8):1105-1122 